Retos, II (2I), 202 I

\title{
Crecimiento económico y la teoría de la eficiencia dinámica
}

\section{Economic growth and the dynamic efficiency theory}

Dr. Armando José Urdaneta-Montiel es profesor e investigador titular de la Universidad Metropolitana (Ecuador) (aurdaneta@umet.edu.ec) (https://orcid.org/0000-0002-9825-9453)

Dr. Emmanuel Vitorio Borgucci-García es profesor e investigador de la Universidad del Zulia (Venezuela) (emmanuelborgucci@gmail.com) (https://orcid.org/0000-0002-4078-006X)

Mg. Bladimir Jaramillo-Escobar es profesor e investigador de la Universidad de Guayaquil (Ecuador) (bladimir.jaramilloe@ug.edu.ec) (https://orcid.org/0000-0001-9984-813X)

\begin{abstract}
Resumen
Esta investigación analiza el crecimiento económico desde la teoría de la eficiencia dinámica, utilizando un indicador global de competitividad y uno de libertad económica global, partiendo de la hipótesis de que mayor libertad económica se traduce en mayor competitividad y crecimiento económico. La eficiencia dinámica sustentada en autores de la economía austriaca pretende explicar cómo el incremento en la rentabilidad y productividad en la producción de bienes y servicios, depende principalmente de la creatividad empresarial. Desde el punto de vista metodológico, el estudio es descriptivo, correlacional y prospectivo, se empleó datos de panel de las 20 economías más grandes del continente americano; también analiza los principales indicadores macroeconómicos, la calidad de las instituciones, salud, educación primaria, infraestructura y el grado de innovación empresarial, correlacionado con variables que miden el nivel de libertad para hacer negocios, presión fiscal, tamaño del gobierno, seguridad jurídica; se mide la competitividad por medio de los factores que determinan la productividad de una economía. Entre los principales resultados se encontró que el índice de libertad económica y el PIB per cápita, muestran una relación causal bidireccional en el sentido de Granger, develando con ello una relación de endogeneidad entre ambas variables. Se demostró el grado de cointegración, causalidad y explicación de la competitividad y la libertad económica con el crecimiento económico.
\end{abstract}

\begin{abstract}
This research analyzes economic growth from the theory of dynamic efficiency, using a global indicator of competitiveness and one of global economic freedom, starting from the hypothesis that greater economic freedom translates into greater competitiveness and economic growth. The dynamic efficiency supported by authors of the Austrian economy aims to explain how the increase in profitability and productivity in the production of goods and services depends mainly on business creativity. From the methodological point of view, the study is descriptive, correlational and prospective, using panel data from the 20 largest economies in the American continent. The study analyzes the main macroeconomic indicators, the quality of institutions, health, primary education, infrastructure and the degree of business innovation, correlated with variables that measure the level of freedom to do business, fiscal pressure, size of government, security Legal, competitiveness is measured through the factors that determine the productivity of an economy. Among the main results, it was found that the index of economic freedom and the GDP per capita show a bidirectional causal relationship in the Granger sense, thus revealing an endogenity relationship between both variables. The degree of cointegration, causality and explanation of competitiveness and economic freedom with economic growth was demonstrated.
\end{abstract}

\section{Palabras clave I keywords}

Competitividad, libertad económica, función empresarial, crecimiento económico, eficiencia dinámica, innovación, productividad.

Competitiveness, economic freedom, business function, and economic growth, dynamic efficiency, innovation, productivity.

Cómo citar: Urdaneta-Montiel, A.J., Borgucci-García, E.V., y Jaramillo-Escobar, B. (2021). Crecimiento económico y la teoría de la eficiencia dinámica. Retos Revista de Ciencias de la Administración y Economía, 11(21), pp. 93-116. https://doi.org/10.17163/ret.n21.2021.06 


\section{Introducción}

Este estudio plantea una metodología que permita explicar el crecimiento económico desde la teoría de la eficiencia dinámica, utilizando un indicador global de competitividad y uno de libertad económica global. El indicador de competitividad mide la calidad de las instituciones, infraestructura, ambiente macroeconómico, salud y educación primaria, todo ello como requerimientos básicos; así se consideran una serie de indicadores que potencian la eficiencia económica como son la educación superior y capacitación para el trabajo, eficacia del mercado de bienes y trabajo, desarrollo de los mercados financiero, disponibilidad tecnológica, y del tamaño del mercado; finalmente se asumen el descubrimiento empresarial y sofisticación de los negocios como elementos fundamentales de la innovación. El indicador de libertad económica mide el nivel de libertad para hacer negocios y comercializar, el nivel de presión fiscal y tamaño del gobierno, el nivel de independencia de la política monetaria y del ente rector que la instrumenta, es decir, libertad monetaria; así mismo la seguridad jurídica para la inversión o libertad de inversión, garantías jurídicas al derecho de propiedad, bajos niveles de discrecionalidad en el funcionamiento de las instituciones del Estado o libertad de corrupción, flexibilidad laboral o libertad laboral en la consumación y disolución de los contratos de trabajo o relaciones laborales entre patrono y trabajador. Se plantea la hipótesis de que mayor libertad económica se traduce en mayor competitividad y crecimiento económico. Ahora bien, desde el enfoque de la escuela austriaca de la economía ambas variables son producto de la función empresarial y es generado por procesos de eficiencia dinámica dentro del libre mercado.

Por esta razón, el trabajo se subdividirá en: 1) un resumen breve de las características de la investigación y los fundamentos teóricos que sustentan al estudio; 2) la metodología asumida para integrar la teoría con los referentes empíricos; 3 ) los resultados de un modelo matemático de aproximación de crecimiento económico desde la competitividad y libertad económica de los respectivos países sujetos a estudio; y 4) las conclusiones del caso.

\subsection{El libre mercado, la función empresarial y la eficiencia dinámica}

La competitividad, libertad económica y crecimiento son conceptos muy antiguos sobre todo a partir de la rebelión contra el mercantilismo en el siglo XVII, donde liberales como Thomas Le Gendre, acuñaron la frase laissez-faire (dejar hacer) ${ }^{1}$ (Rothbard, 2012). Posteriormente vendría a finales del siglo XVIII el gran pensador y estadista Anne Robert Jaques Turgot. Dicha doctrina económica planteaba que el comercio solo puede florecer y subsistir cuando los mercaderes son libres para procurarse las mercancías que necesitan en los lugares en las que se venden al precio más bajo (Rothbard, 2012) y Mises (1983[1944]).

Por ello, Schumpeter (1983) caracterizó al mercado como el lugar donde se desarrollan procesos competitivos intertemporales, donde el riesgo económico está siempre presente en la fijación de los precios de los bienes y servicios, además de otras cosas

1 Según Fergunson (1979[1938]) y Schumpeter (1994[1954]) la frase se le atribuye acertadamente o equivocadamente a Vincent de Gournay (1712-1759). La oración sería: "Laissez faire laissez passer le monde va de lui-même”. Según Schumpeter (1994[1954]), Gournay tuvo dos grandes aportes a la economía y en concreto a la libertad económica: 1) fue junto a Mirabeau uno de los principales divulgadores de la obra de Richard Cantillon (1680-1734), ya que consideraron que era una obra sistemática y didáctica que la convirtió en un gran antecedente a la obra de Quesnay; y 2) “...su aportación a la formación económica de Anne Robert Jacques Turgot (1721-1781)” (Schumpeter, 1994[1954], p. 289). 
más allá de los contratos de compra-venta. De acuerdo con Schumpeter (1983), los procesos de mercado suministran un caso relevante para distinguir claramente entre lo que el autor denomina como "corriente circular" y "desenvolvimiento" (Entwicklung).

En lo referente al desenvolvimiento, Schumpeter (1983) lo caracteriza de la siguiente manera: 1) alteraciones del sistema económico provenientes de la esfera económica; 2) surgen del crecimiento de la actividad económica, la población o la riqueza; 3 ) no se refiere a procesos de adaptación del sistema económico, por cuanto está relacionado a factores exo-económicos; 4) como un proceso que reposa sobre el desenvolvimiento precedente; 5) están asociadas a alteraciones espontáneas y discontinuas de la corriente circular; 6) por la puesta en práctica de nuevas combinaciones de los elementos que sirven de materiales para la producción, los métodos de producción, los sistemas de empaque, los sistemas de etiquetado, los sistemas de comercialización o los mercados a los que se destinará a satisfacer las necesidades; y 7) ser el resultado de la competencia no pura.

Dentro de la corriente circular y el desenvolvimiento es donde las empresas y la función empresarial actúan. Así, para Knight (1964[1921]): "Nosotros vivimos solamente por el conocimiento en torno al futuro; mientras los problemas de la vida, o de la conducta al menos, surgen del hecho de que solo conocemos una pequeñísima porción del futuro" (p. 199). El hombre y su conciencia, dice Frank Knight, se enfrenta al entorno y se adapta constantemente a él. El hombre reacciona a la "imagen" de un futuro que puede ser, entre otras cosas, la situación futura de los negocios; y por el sentido común, la imagen, es a la vez presente y operativa; la imagen es espontánea y mira hacia adelante. Para Frank Knight, el rol de la conciencia es dar al organismo el conocimiento del futuro y basándose en René Descartes: "Percibimos el mundo antes de que podamos reaccionar ante él, y reaccionamos no a lo que percibimos, sino siempre a lo que inferimos" (Knight, 1964[1921], p. 201).

Pero el desenvolvimiento requiere un lugar donde se desarrollen las combinaciones y el actor que las lleva a cabo. De esta forma Schumpeter expresa lo siguiente: "Llamamos "empresa" a la realización de nuevas combinaciones, y "empresarios" a los individuos encargados de dirigir dicha realización” (Schumpeter, 1983, p. 84).

Leibenstein (1969), la función empresarial serían todas aquellas acciones que buscan reducir las ineficiencias del ciclo organizacional de la empresa. Esas ineficiencias provienen de la incompleta especificación de los contratos y las lagunas de conocimiento tanto del ciclo organizacional como del mercado.

Para Huerta de Soto (2010): "La función empresarial es la capacidad típicamente humana para darse cuenta de las oportunidades de ganancias que surgen en el entorno actuando en consecuencia para aprovecharse de ellas" (p. 110). Por su parte, Kirzner (1979), el entrepreneurship no funciona siempre en condiciones de equilibrio al estilo neoclásico. El entrepreneurship en ocasiones puede enfrentar situaciones de ganancias y situaciones de pérdida, de situaciones no equilibrantes.

Estas ideas tienen de base común las propuestas de Mises (1983[1944]) y Hayek (1978):

Aquellos deseosos de obtener ganancias siempre están buscando una oportunidad. Tan pronto como descubren que la relación de los precios de los factores de producción con los precios anticipados de los productos parece ofrecer tal oportunidad, intervienen. Si su valoración de todos los elementos involucrados fue correcta, obtienen una ganancia. (Mises, 1983[1944], pp. 31-32)

Pero para Mises (1983[1944]) la naturaleza de las ganancias se encuentra en el hecho de que el mundo real no es estacionario, sino cambiante y en ese sentido el autor expone: 
Pero el mundo actual es un mundo en permanente cambio. Las cifras, los gustos y las necesidades de la población, el suministro de factores de producción y los métodos tecnológicos están en un flujo incesante. En tal estado de cosas, es necesario un ajuste continuo de la producción al cambio de condiciones. Aquí es donde entra el emprendedor. (Mises, 1983[1944], p. 31)

Ahora bien, el libre mercado y con él la competencia se abordan desde la perspectiva de la teoría de eficiencia dinámica; pretende explicar cómo el incremento en la rentabilidad y productividad en la producción de bienes y servicios, depende principalmente de la creatividad empresarial y la especulación ${ }^{2}$ en las actividades comerciales, evitando el despilfarro de los recursos dados de acuerdo con la eficiencia estática (Huerta, 2010). La dimensión dinámica de la eficiencia económica parte de que los recursos pueden variar como resultado de procesos creativos junto a la coordinación dentro de la empresa como consecuencia de la búsqueda incesante de descubrir y superar los desajustes sociales (Huerta, 2010).

Por esta razón, la función empresarial siempre genera nueva información (Huerta, 2010). Esto ocurre por los desajustes sociales que genera el mercado que, a su vez, significan nuevas posibilidades de negocios y ganancias. Dicha información se trasmite principalmente a través del sistema de precios en los diversos mercados del sistema económico.

Equilibrio económico no es igual que la teoría del equilibrio general. Los orígenes del primero se originan en la escolástica tardía (Schumpeter, 1994[1954]). Desde Santo Tomás, el equilibrio si se quiere individual parte del respeto a la idea de bien común o bien justo. El equilibrio, siguiendo a Aristóteles (1992) es una proporción siguiendo las reglas de la justicia conmutativa. La primera visión amplia del proceso económico vino de la mano de san Antonio Pierozzi (1389-1459) y Gabriel Biel (1425-1495). Ya en la escolástica tardía el precio justo se identificó no solamente con el precio competitivo normal, sino con cualquier precio competitivo. Con Pietro Verri (1728-1797) la visión de equilibrio se basó en cálculos del placer y el dolor al estilo de Jeremy Bentham. Pierre le Pesant sieur de Boisguillebert (1646-1714), el equilibrio es de magnitudes económicas interdependientes, pero desde el punto de vista del consumo (Schumpeter, 1994[1954], p. 259). La primera exposición matemática del equilibrio se debe a Achille Isnard. Si bien con Charles Devenant, Josiah Child y John Pollexfen construyen relaciones entre magnitudes económicas, es con el Tableau Cantillon-Quesnay que se llegó al primer método para exponer la naturaleza del equilibrio económico visto como agregados sociales. El proceso antes trazado fue continuado por Smith (1982[1776]), al estudiar los componentes del precio (costes e ingresos: salarios, renta y beneficio), estableció una primitiva interdependencia de las magnitudes que conforman el sistema económico. Hay que hacer mención especial a Ferdinando Galiani quien expuso el equilibrio económico desde una perspectiva de largo plazo (Schumpeter, 1994 [1954]). Dentro

2 Del sustantivo latín speculātio y del verbo latin specuiari, que significa registrar, mirar, observar con atención una cosa para reconocerla y examinarla y speculātor, observador, espía, explorador. La especulación comercial se trata de comprar (o vender ciertos productos a un determinado precio para revenderlos (o volverlos a comprar) a un precio a mayores o menores precios que se dan en otras circunstancias de tiempo y lugar con la finalidad de obtener un lucro. Es decir, el negocio especulativo consiste en conocer la forma en que mercado trabaja, por medio de oferentes y demandantes con la finalidad de sacar provecho del arbitraje. Las condiciones necesarias para que se presente son: 1) que los bienes objeto del negocio se negocie en diferentes mercados, para localizar sus variaciones diferenciales de precios; 2) el mercado no esté en condiciones de compensar por su cuenta tales variaciones. En el caso de la Economía, la especulación es el esfuerzo que se realiza para sacar provecho, del conocimiento del mercado del producto de interés del especulador, sobre el sendero que tomará su precio (Salvat, 1972). 
de los clásicos hay que destacar a: Jean-Baptiste Say (1767-1832) (2001[1841]), cuyas ideas de equilibrio económico unieron los aportes de Richard Cantillon (2002[1959]) y Jacques Turgot (Aramburo-González, 1998) con Léon Walras. Con Nicolás Barbón (1640-1698), el equilibrio económico se extiende al comercio internacional y con John Stuart Mill (1806-1873) (1985[1848]) cobra vigencia el análisis estático y la idea de estado estacionario insistentemente empleada por David Ricardo (1772-1823) (1973) y sir Thomas Robert Malthus (1766-1834) (1977).

En este sentido, la teoría de la eficiencia dinámica se desmarca del concepto de equilibrio económico y particularmente del walrasiano y sus variantes. Para Huerta de Soto (2010), la eficiencia dinámica se sustenta en la creatividad, el descubrimiento y la coordinación organizacional. Consiste en adaptar fines y medios dentro de desajustes crónicos en cualquier parte del sistema económico relacionado con la organización y sus variables económicas más importantes. Necesariamente, no se llegará a un punto de indiferencia (límite paretiano de eficiencia) en una función de posibilidades de producción, lo que implicaría subutilización de recursos, por cuanto lo que interesa es que la empresa pueda cazar oportunidades de ganancias y con ello de expansión de su función de posibilidades. En esa idea de cazar oportunidades, Kirzner (1979) propone la idea de que los empresarios realizan actos empresariales en busca de ganancias en un contexto donde el fracaso es una posibilidad (no estadística) y en donde se presenta un aprendizaje del comportamiento de las variables que afectan al negocio. Esa obtención de ganancias se convierte en un mecanismo de transmisión de información y de coordinación de las actividades de todo un sector económico.

Ahora bien, desde el punto de vista del contexto, la empresa se encuentra que no puede conocer completamente el conjunto de fines y medios de los cuales tomará decisiones. Esta incompletitud en el abarcar todas las posibilidades del contexto se debe a que la variedad de negocios es indeterminada, al menos, con facilidad (Rothbard, 1979). Por lo tanto, tomar decisiones asumiendo determinada función genérica de utilidad tendería a subestimar o sobreestimar la realidad de los negocios. Para Rothbard (1979), un elemento que puede ayudar a mejorar la eficiencia dinámica es contar con un marco ético adecuado en que al menos se garantice el derecho a la propiedad y que se garantice la posibilidad de ejecutar acuerdos voluntarios. En palabras de Demmert y Klein (2003) sería: "Un régimen de libertad —impuestos bajos, propiedad privada segura, restricciones mínimas sobre acuerdos voluntarios- podría promover todo tipo de descubrimiento y mejorar la alineación entre la oportunidad individual y el mejoramiento social" (p. 299).

Finalmente, Joseph A. Schumpeter propone, aparte del papel del empresario expuesto más arriba, la idea de destrucción creativa (Creative destruction) y en ese sentido:

El impulso fundamental que pone y mantiene en movimiento el motor del capitalismo proviene de los nuevos bienes de consumo, los nuevos métodos de producción y transporte, los nuevos mercados, las nuevas formas de organización industrial que crean las empresas capitalistas. (Schumpeter, 2008[1942], p. 83)

No obstante, esa destrucción creativa y en consecuencia de "costos hundidos" para muchas organizaciones no se presenta en un mercado competitivo perfecto ni en situaciones de equilibrio automático e instantáneo. Esa destrucción se encuentra en el llamado desenvolvimiento (Entwicklung) y que ese proceso de destrucción que presupone un proceso de creación es de carácter sorpresivo. Por lo que respecta a Leibenstein (1966), las relaciones económicas entre agentes pueden llevar a contratos que no consideren todas las situaciones posibles entre oferentes y demandantes. Es decir, la generación de vacíos, áreas de incertidumbre o de indeterminación en situaciones con- 
cretas puede llevar a tomar decisiones erróneas en las empresas. Las malas decisiones se pueden agravar en un contexto que permita la existencia de "loophole minning" (Kane, 1981) o problemas de selección adversa (Adverse Selection) (Akerlof, 1970). El resultado final de contexto en contra y malas decisiones es la ineficiencia organizacional.

Una de las cosas que se deben considerar de lo anteriormente expuesto es que la idea de equilibrio automático, instantáneo, simultáneo y perenne ${ }^{3}$ de los economistas neoclásicos es un concepto alejado de la realidad de la economía debido a que: 1) se parte de una versión del individualismo ontológico y metodológico que supone que los agentes económicos tienen todo el conocimiento para tomar sus decisiones económicas (Hayek, 1989); 2) se parte del supuesto "heroico" (Bunge, 1999) de que la economía es un sistema cerrado tanto en sus relaciones con el gobierno como del resto del mundo; 3) la racionalidad se entiende tanto como la maximización inevitable de la utilidad y beneficio total lo cual, según Morgenstern (1972), implica que personas y empresas controlen todas las variables de las que depende el máximo; 4) el mercado es perfectamente competitivo y que el precio expresa ese equilibrio, aunque en el largo plazo el precio sea igual al costo medio unitario lo cual hace difícil explicar la ganancia (Samuelson et al., 1983); 5) los agentes y sus deseos son mutuamente excluyentes, son variables aleatorias; 6) la existencia de la "mano invisible" como mecanismo de autorregulación del sistema.

Ahora bien, cuando la interacción de la función empresarial y la competitividad se llevan al plano macroeconómico y en concreto del crecimiento económico desde la perspectiva austriaca se debe considerar el aporte de Garrison (2005) en el sentido de considerar dos problemas cruciales: el problema de si el mercado funciona y el enfoque en que se debe abordar desde el trabajo o desde el capital. En este sentido, en el primer problema se considera la cuestión de si las decisiones descentralizadas desemboquen en coordinación o descoordinación macroeconómica. La orientación analítica, por su parte tiene que ver sobre la preferencia de abordar la coordinación en el estudio del mercado de trabajo o de capitales. Tanto John M. Keynes y Milton Friedman se enfocan en el estudio del mercado de trabajo, pero mientras que el primero parte de que el mercado no funciona de manera adecuada, el segundo considera que sí lo hace. Por su parte, Friedrich von Hayek considera que los mercados, al funcionar sin interferencia del gobierno y estudiando el problema económico desde el factor capital, produce mejores decisiones económicas.

En consonancia, se han elaborado indicadores que por una parte enfatizan los resultados del proceso económico como el producto interno bruto per cápita con la productividad del trabajo y por otra evalúan los factores determinantes. En lo referente

3 Cuando se habla de la teoría del equilibrio general, se parte inicialmente de los trabajos de Cournot (1863) y seguidamente de Walras (1987[1874]). Las versiones de Walras son modelos de largo plazo y se interesó por saber cuándo el equilibrio era definitivo y estable. El equilibrio para Walras implicaba un proceso conocido como "Tâtonnement", en donde un rematador anunciaba un precio y los agentes respondían con sus cantidades de oferta y demanda que estaban dispuestos a comprometerse. Cuando se presentaba una $\mathrm{n}$ coincidencia de precios, o de producía o se transaba en los mercados envueltos. Posteriormente surgieron los modelos de Kenneth Arrow, Gerard Debreu y Lionel W. McKensie en la década de los 50. En años posteriores, tanto los New Keynesians como los economistas de nueva macroeconomía clásica (sin olvidar de que cuentan, por ejemplo con la teoría delos ciclos reales), aceptan que los modelos macroeconómicos deberían ser de equilibrio general tanto en el mundo de la competencia perfecta con salarios y precios flexibles, sino que se emplea lo que se conoce como los modelos dinámicos de equilibrio general estocástico (Dynamic Sthocastic General Equilibrium o DSGE) para estudiar los efectos a corto plazo de políticas alternativas en contextos de competencia imperfecta, tanto en el mercado de trabajo como el de productos (Woodford, 2008). 
a los segundos, se han contemplado cuatro grandes bloques: infraestructuras y accesibilidad, recursos humanos, innovación tecnológica y entorno productivo. (Cuadernos Fundación BBVA, 2008). A tenor de ello, DeLong (2003) y la Comisión Europea (1999) en su sexto informe periódico acerca de la situación económica y social apoya la idea de que la competitividad es importante para alcanzar el crecimiento económico, del ingreso per cápita con el concurso de la acumulación y uso eficiente del capital físico, humano, intelectual y tecnológico obtenidos por medio del mecanismo del libre mercado, donde las compañías, industrias, regiones, naciones, regiones supranacionales compiten con niveles relativamente altos de ingresos y empleo.

Los factores determinantes del crecimiento del ingreso per cápita desde la competitividad económica serían: 1) el inventario de capital fijo productivo en relación con el empleo calificado; 2) infraestructuras de transportes, comunicaciones y facilidades productivas; 3) la existencia de departamento de Investigación y Desarrollo en las empresas; 4) aspectos de carácter exoeconómico tales como: localización geográfica, carácter de las relaciones laborales, políticas públicas orientadas a la producción y la productividad, entre otros aspectos (Cuadernos Fundación BBVA, 2008).

Los elementos causales de la dicha competitividad serían una serie de indicadores relacionados a los precios y costes relativos como son: el comportamiento en el tiempo de las exportaciones netas per cápita a escala mundial o continental; los resultados favorables o desfavorables de la balanza comercial; el grado de penetración de las adquisiciones de un país desde el resto del mundo en los mercados regionales o locales y el comportamiento de los llamados indicadores de ventajas comparativas reveladas (Cuadernos Fundación BBVA, 2008).

Por tal motivo, la competitividad económica puede contemplarse desde dos visiones: 1) la externa, basada en las capacidades y potencialidades de la sostenibilidad de la producción, sustentada en costos medios unitarios comparativos bajos para participar con fuerza en el resto del mundo (Cuadernos Fundación BBVA, 2008); y 2) la agregada, que sugiere una perspectiva de oferta y demanda agregada de la competitividad, que centra su atención en la productividad por trabajador.

Así mismo, Traverso et al. (2017) señalan que la competitividad (medido por el Global Competitivines Report producido por el World Economic Forum) es una variable altamente asociada con el crecimiento económico per cápita. Es decir, las economías más competitivas son las que más crecen en términos per cápita. No obstante, se debe considerar además la libertad económica como un indicador que explica tanto la competitividad como el crecimiento económico. La afirmación antes realizada se sustenta en los postulados de Rothbard (2001) quien señala:

Libre competencia, es la aplicación de la libertad al ámbito de la producción: libertad de comprar, vender y transformar los propios bienes sin intromisión violenta de ningún poder externo. Esto se debe a que un régimen de competencia libre, la satisfacción de los consumidores tiende a maximizarse, dentro de las condiciones naturales existentes. Quienes hacen mejor sus previsiones tienen posibilidad de surgir como empresarios destacados, y si alguno ve una oportunidad no aprovechada, está en libertad para sacar ventaja de su mejor capacidad especulativa. (p. 70)

Por lo antes expuesto, el crecimiento económico se sustenta en la triada compuesta por la función empresarial, la competitividad y la libertad económica desde los principios de la escuela austriaca de economía como muestra la Figura 1. En consecuencia, este trabajo utilizará como variable explicativa del crecimiento económico el índice de libertad económica e índice global de competitividad. 


\section{Figura 1. Triángulo del crecimiento económico}

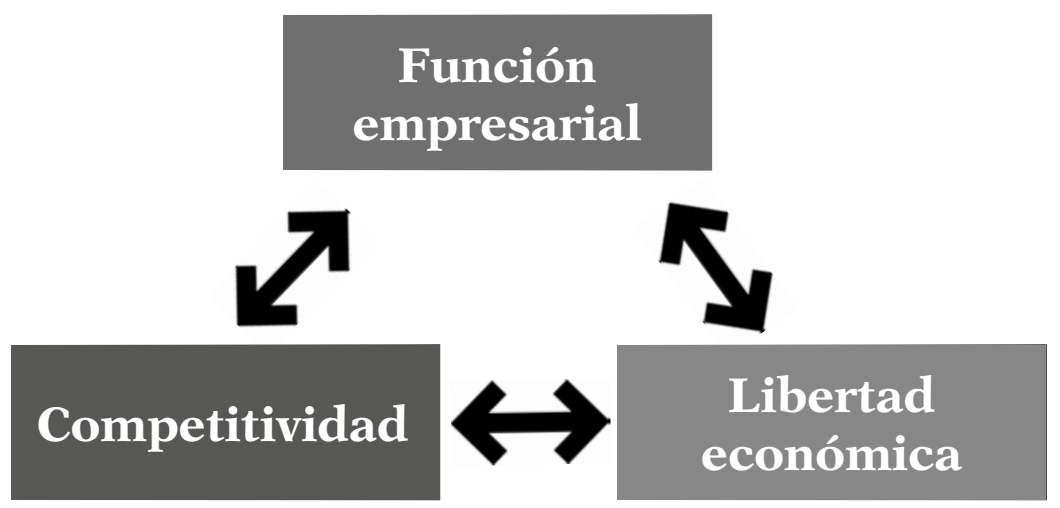

Fuente: Elaboración propia (2019).

Ahora bien, existen posturas que dan cuenta de la relación entre el crecimiento económico, la productividad y la competitividad de la economía. Schumpeter (1983) establece una diferencia entre su concepto de "Desenvolvimiento económico" y el crecimiento de la economía:

Tampoco se llamará aquí proceso de desenvolvimiento al mero crecimiento de la economía, reflejado por el de la población y la riqueza. Pues no representa fenómenos cualitativamente diferentes, sino solamente procesos de adaptación, de la misma clase que los cambios en los datos naturales. (Schumpeter, 1983, p. 74)

Y agrega lo siguiente para explicar la cita anterior: "Hacemos esto porque estos cambios son pequeños anualmente y no significan, por tanto, dificultades para la aplicación del método estático. Sin embargo, su existencia es a menudo una condición de desenvolvimiento, en nuestro sentido" (Schumpeter, 1983, p. 74).

Un autor que relacionó la función empresarial con el incremento del ingreso per cápita fue Leibenstein (1968). Él afirmó que ese crecimiento solo era posible con el campo de los patrones tecnológicos de las empresas mediante la creación de nuevos productos, nuevas materias primas, nuevas formas organizacionales y nuevos conocimientos. De esta manera, Harvey Leibenstein visualiza al empresario como el insumo, el elemento clave y primer inspirador del proceso de crecimiento. Para Kirzner (1985), una vez que el empresario es tomado en cuenta en el crecimiento económico, el énfasis se centra en el descubrimiento de nuevas estructuras de fines y medios. Esta visión permite dar cuenta de los cambios en el conjunto de insumos y de las relaciones entre estos y los resultados de los procesos organizacionales (incluyendo los productivos). Para comprender el proceso de crecimiento económico se debe abordar el problema de la asignación de recursos desde la óptica de los procesos de descubrimiento.

Por su parte, Baumol (1990), parte de la sencilla idea de que mientras el total de empresarios es más o menos fijo, su contribución al crecimiento podría variar en mayor medida. El empresario podría o no contribuir con la renta per cápita dependiendo de los ingresos relativos que una sociedad ofrece. Es decir, la contribución del empresario dependerá de lo estable de las reglas de juego que una sociedad/gobierno ofrecen, ya que eso influye en la estructura del sistema de recompensas. Esa estructura de recompensas, a su vez, incide sobre el patrón de asignación de recursos. En resumen, para Baumol (1990), existe un fuerte vínculo entre el grado en el cual una economía gratifica socialmente la actividad emprendedora productiva y la prosperidad de ese sistema económico. La mayoría de los estudios confirman la relación positiva entre la actividad emprendedora y el crecimiento económico (Córdova et al., 2020). 
También existen otros elementos que aportan al crecimiento económico como el grado de apertura económica de un país con el resto del mundo (Molero et al., 2020).

De esta manera, las relaciones entre las variables de la triada antes expuesta podrían depender de: 1) el grado de complejidad del trabajo realizado y la calidad en la formación de mano de obra calificada a escala mundial; 2) la intervención con ventajas en el comercio exterior; 3) las disparidades en los ingresos por habitante; 4) las desproporciones sociodemográficas; 5) la velocidad en el crecimiento económico; 6) la accesibilidad y disponibilidad de factores productivos; 7) el clima social imperante y sus efectos en el desenvolvimiento del sistema político; y 8) factores asociados al riesgos y los costos de cobertura entre otros factores (Cadavid \& Franco, 2006).

\section{Materiales y método}

Desde el punto de vista metodológico, el estudio es descriptivo, correlacional y prospectivo. Se empleó datos de panel de las 20 economías más grandes del continente americano, agrupadas en cuatro clústeres: 1. Ecuador, Nicaragua, Argentina, Bolivia y Venezuela; 2. Colombia, Perú, Brasil, El Salvador, Paraguay, Honduras, Guatemala y República Dominicana; 3. Chile, México, Uruguay, Costa Rica y Panamá; 4. Estados Unidos y Canadá. Para las pruebas de estadísticas se utilizaron los programas Eviews 10.0 y SPSS 24.0, las variables objeto de estudio fueron estandarizadas con puntuaciones estándar (número de desviaciones estándar que hay por encima o por debajo de la media del ranking e índice de competitividad global publicado por el Foro Económico Mundial). ${ }^{4}$ La descripción se refiere al proceso de perfilar cómo es la relación entre índice de competitividad y el índice global de libertad con el crecimiento económico (Marczyk et al., 2005). La investigación correlacional intenta determinar si existe cierto grado de asociación estadística entre los dos índices presentados anteriormente con el crecimiento económico. Finalmente es prospectiva, porque de verificarse la correlación, entonces es posible adelantar aproximaciones sobre el comportamiento futuro de la variable, considerando ciertas condiciones y restricciones (Marczyk et al., 2005).

Por lo tanto, este estudio se enmarca en el enfoque de los índices de competitividad global (en adelante ICG) del World Economic Fórum (en adelante WEF). Se mide la competitividad por medio de los factores que determinan la productividad de una economía y concretamente los de los niveles de ingreso y crecimiento a largo plazo. Luego, el ICG evalúa la productividad a través de los denominados 12 "pilares", prestando atención en la participación del capital humano, la creatividad productiva, la capacidad de recuperación ante coyunturas desfavorables y la flexibilidad en los sistemas organizacionales vistos como factores que guían el éxito económico-financiero. Esto son la seguridad, los derechos de propiedad, el capital social, los controles y balances, el desempeño del sector público y la administración corporativa; la calidad y la extensión de la infraestructura de transporte y la infraestructura para los servicios públicos; adopción de tecnologías de la información y comunicación (TIC), el nivel de inflación y la sostenibilidad de la política fiscal; estudia la esperanza de vida ajustada a la salud, es decir el número promedio de años que se espera que un recién nacido pueda vivir si cuenta con buena salud; el nivel general de habilidades de la fuerza laboral y la cantidad y calidad de la educación.

Así mismo, estudia el grado en que un Estado nacional brinda oportunidades equitativas de libre acceso a sus mercados; analiza las formas en que se reorganizan y

4 Publicado anualmente desde 1979 abarca 140 países; el índice y ranking de libertad económica creado en el año 1995, incluye a 180 naciones, y publicado desde entonces anualmente por la Fundación Heritage y el Wall Street Journal. 
aprovechan los recursos humanos; examina lo diversificado y profundo de los mercados monetario financiero; los límites de tolerancia de la economía ante riesgos financieros y económicos. Seguidamente, estudia la dimensión en términos demográficos y de poder adquisitivo de los mercados a los que podrían incursionar las empresas. ${ }^{5}$ Finalmente, analiza el escenario para la producción de conocimientos científicos convertibles en investigación aplicada a procesos, bienes y servicios para lograr una mayor competitividad en términos de calidad y cantidad (Centro de Estudios sobre la Cuenca del Pacífico, 2018).

\section{Resultados}

Este estudio comenzó con la realización de la prueba de causalidad de Granger, donde la probabilidad del estadístico F cuya regla de decisión es rechazar Ho: No existe causalidad entre las variables, siendo la regla de decisión: Si Prob $<0,05$ se Rechaza Ho. En este sentido, se pudo constatar que el índice de competitividad causa en términos de Granger (antecede) al PIB per cápita; más el PIB per cápita no causa en términos de Granger (antecede) al índice de competitividad tal como se muestra en la Tabla 1. Por su parte, el índice de libertad económica y el PIB per cápita, muestran una relación causal bidireccional en el sentido de Granger, develando con ello una relación de endogeneidad entre ambas variables. También es de destacar la no causalidad bidireccional en términos de Granger entre el índice de libertad económica y el índice de competitividad.

En el análisis del triángulo del crecimiento económico propuesto en la fundamentación teórica del trabajo, que en el caso de las 20 economías más grandes del continente americano sujetas a estudio, demuestran que el índice de libertad económica es una variable endógena para estimación del PIB per cápita, por lo tanto los rezagos de dicha variable causan un impacto en los valores futuros del PIB per cápita, mientras el índice de competitividad es una variable exógena en la estimación del PIB per cápita.

\section{Tabla 1. Prueba de causali2dad de Granger}

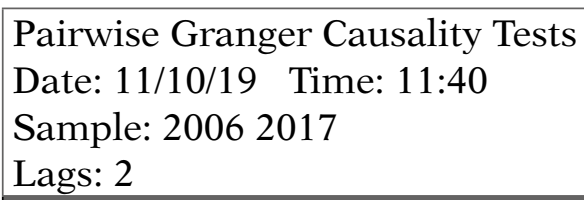

\begin{tabular}{|c|c|c|c|}
\hline Null Hypothesis: & Obs & F-Statistic & Prob, \\
\hline $\begin{array}{l}\text { INDICE_DE_LIBERTAD_ECONOMICA does not Granger Cause } \\
\text { INDICE_DE_COMPETITIVIDAD }\end{array}$ & 200 & 237.105 & 0,0961 \\
\hline $\begin{array}{l}\text { INDICE_DE_COMPETITIVIDAD does not Granger Cause } \\
\text { INDICE_DE_LIBERTAD_ECONOMICA }\end{array}$ & & 204.678 & 0,1319 \\
\hline $\begin{array}{l}\text { PIB_PER_CAPITA does not Granger Cause } \\
\text { INDICE_DE_COMPETITIVIDAD }\end{array}$ & 200 & 282.044 & 0,062 \\
\hline $\begin{array}{l}\text { INDICE_DE_COMPETITIVIDAD does not Granger Cause } \\
\text { PIB_PER_CAPITA }\end{array}$ & & 365.471 & 0,0277 \\
\hline $\begin{array}{l}\text { PIB_PER_CAPITA does not Granger Cause } \\
\text { INDICE_DE_LIBERTAD_ECONOMICA }\end{array}$ & 200 & 461.399 & 0,011 \\
\hline $\begin{array}{l}\text { INDICE_DE_LIBERTAD_ECONOMICA does not Granger Cause } \\
\text { PIB_PER_CAPITA }\end{array}$ & & 483.245 & 0,0089 \\
\hline
\end{tabular}

Fuente: Eviews 10.0 (2019).

5 Sus valores se obtienen con la suma del consumo, la inversión y las exportaciones. Esta evalúa la actitud del sector privado para generar y adoptar nuevas tecnologías e insertarlas en su proceso productivo y organizacional. 
Así mismo se realizó la prueba de cointegración de residuos de Pedroni para datos de panel, tomando un solo rezago, evidenciándose que 7 de los 11 estadístico de prueba muestran una probabilidad $($ Prob $<0,05)$ con lo cual se demuestra que las variables sujetas a estudio, es decir índice de competitividad, índice de libertad económica y PIB per cápita están cointegradas, esto es tienen una relación a largo plazo. Por lo tanto, la hipótesis nula es no cointegración, La regla de decisión es rechazar H0 si Prob $<0,05$ en este caso 7 de las 11 pruebas rechazan $\mathrm{H} 0$ es decir las variables están cointegradas porque la Prob. $<0,05$ tal como se muestra en la Tabla 2.

El modelo de análisis factorial, afirma que las covarianzas en un conjunto de variables observables $\mathrm{X}_{1}, \mathrm{X}_{2}, \ldots, \mathrm{X}_{\mathrm{n}}$ en términos de un reducido número de factores comunes que son latentes no observadas, se presentan en su forma desarrollada como un sistema de ecuaciones lineales en 1 (OECD \& JRC, 2008; Timm, 2002; Peña, 2002).

$$
\begin{aligned}
& \mathrm{X}_{1}-u_{1}=\lambda_{11} f_{1}+\lambda_{12} f_{2}+\ldots+\lambda_{1 \mathrm{k}} f_{\mathrm{k}}+e_{1} \\
& \mathrm{X}_{2}-u_{2}=\lambda_{21} f_{1}+\lambda_{22} f_{2}+\ldots+\lambda_{2 \mathrm{k}} f_{\mathrm{k}}+e_{2} \\
& \text { - } \cdot \text { ・ } \\
& \mathrm{X}_{\mathrm{i}}-u_{\mathrm{i}}=\lambda_{\mathrm{i} 1} f_{1}+\lambda_{\mathrm{i} 2} f_{2}+\ldots+\lambda_{\mathrm{ik}} \dot{f}_{\mathrm{k}}+e_{\mathrm{i}} \\
& \cdot \begin{array}{lll}
\cdot & \bullet & \cdot
\end{array} \\
& \mathrm{X}_{\mathrm{n}}-u_{\mathrm{n}}=\lambda_{\mathrm{n} 1} \dot{f_{1}}+\lambda_{\mathrm{n} 2} f_{2}+\ldots+\lambda_{2 \mathrm{k}} \dot{f_{\mathrm{k}}}+e_{2}
\end{aligned}
$$

Donde: Xi representa son las variables observadas obtenidas de datos y que al estandarizarlos tendrán media $\mathrm{E}(\mathrm{Xi})=0$ y $\sigma^{2}=1$ para toda $\mathrm{i}=1,2, \ldots$, p; las $\lambda 11, \lambda 12$, ..., $\lambda \mathrm{k}$ son coeficientes de regresión, que en esta técnica se les denomina ponderaciones de los factores; las $\mathrm{f} 1, \mathrm{f} 2, \ldots, \mathrm{fk}$ son los denominados factores comunes latentes no observadas que se investigan, cada observación media $\mathrm{E}(\mathrm{Xi})=0$ y $\sigma^{2}=1$; finalmente, los residuales $\mathrm{e}_{\mathrm{i}} \mathrm{o}$ las perturbaciones poblacionales observadas de los factores únicos $\mathrm{o}$ específicos (García et al., 2017). 
Tabla 2. Prueba de cointegración de residuos de Pedroni

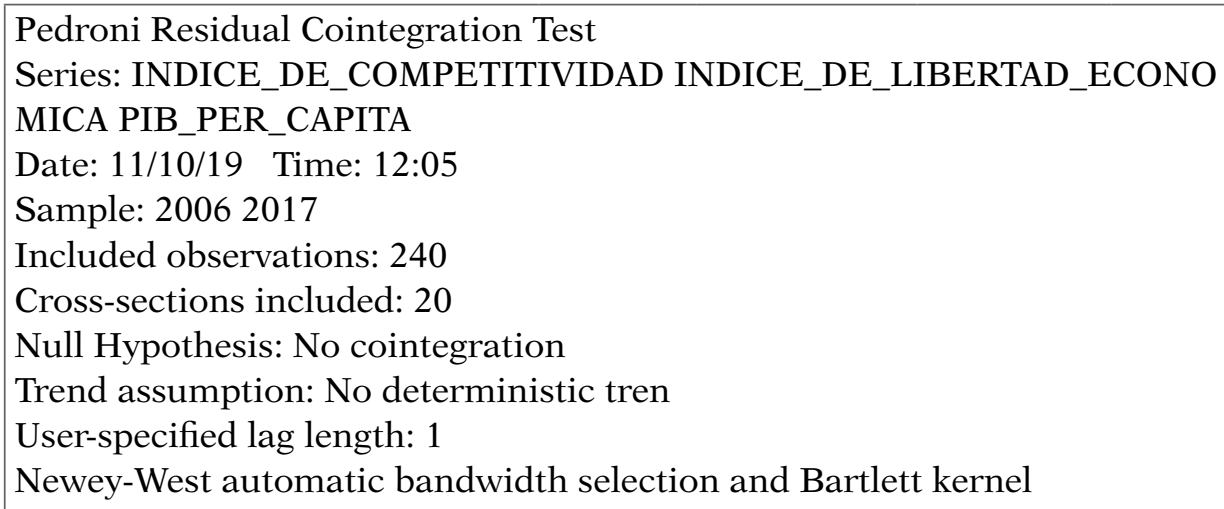

\begin{tabular}{|c|c|c|c|c|}
\hline Panel v-Statistic & 2.344 .857 & 0,0095 & 0,202451 & 0,4198 \\
\hline Panel rho-Statistic & $-0,381267$ & 0,3515 & 0,030367 & 0,5121 \\
\hline Panel PP-Statistic & -3.424 .514 & 0,0003 & -3.087 .803 & 0,001 \\
\hline Panel ADF-Statistic & -3.197 .237 & 0,0007 & -2.954 .698 & 0,0016 \\
\hline
\end{tabular}

Alternative hypothesis: individual AR coefs, (between-dimension)

\begin{tabular}{|c|c|c|c|c|c|}
\hline & & Statistic & Prob. & & \\
\hline Group rho-Statistic & & 1.491 .335 & 0,9321 & & \\
\hline Group PP-Statistic & & -4.141 .125 & 0 & & \\
\hline Group ADF-Statistic & & -4.749 .343 & 0 & & \\
\hline Phil & $\begin{array}{l}\text { Cross s } \\
\text { ips-Pers }\end{array}$ & $\begin{array}{l}\text { ection spec } \\
\text { n results }\end{array}$ & $\begin{array}{l}\text { ific resul } \\
\text { lon-paral }\end{array}$ & (ic) & \\
\hline Cross ID & $\operatorname{AR}(1)$ & Variance & HAC & Bandwidth & Obs \\
\hline ARGENTINA & 0,328 & 0,987572 & 1,175089 & 2 & 11 \\
\hline BOLIVIA & 0,46 & 1,555689 & 1,775483 & 2 & 11 \\
\hline BRASIL & 0,362 & 1,288832 & 1,288832 & 0 & 11 \\
\hline CANADA & $-0,413$ & 1,102382 & 0,862499 & 2 & 11 \\
\hline CHILE & 0,358 & 0,711223 & 0,438157 & 4 & 11 \\
\hline COLOMBIA & 0,081 & 0,179908 & 0,190315 & 1 & 11 \\
\hline COSTA RICA & $-0,301$ & 0,634419 & 0,562050 & 1 & 11 \\
\hline ECUADOR & 0,184 & 1,096583 & 1,379429 & 2 & 11 \\
\hline EEUU & 0,169 & 2,629120 & 2,702221 & 1 & 11 \\
\hline EL SALVADOR & 0,24 & 0,943645 & 0,771107 & 3 & 11 \\
\hline GUATEMALA & $-0,294$ & 1,020709 & 0,607087 & 10 & 11 \\
\hline HONDURAS & $-0,041$ & 1,887664 & 1,014380 & 10 & 11 \\
\hline MÉXICO & 0,112 & 0,354694 & 0,188321 & 6 & 11 \\
\hline NICARAGUA & $-0,032$ & 1,646624 & 1,582661 & 1 & 11 \\
\hline PANAMÁ & $-0,392$ & 0,540932 & 0,495727 & 1 & 11 \\
\hline PARAGUAY & $-0,096$ & 0,511455 & 0,482381 & 2 & 11 \\
\hline PERÚ & 0,097 & 0,246061 & 0,246061 & 0 & 11 \\
\hline REPÚBLICA DOMINICANA & $-0,356$ & 0,234491 & 0,067484 & 10 & 11 \\
\hline URUGUAY & 0,623 & 1,594121 & 2,061241 & 1 & 11 \\
\hline VENEZUELA & $-0,279$ & 1,399358 & 1,030636 & 2 & 11 \\
\hline
\end{tabular}

Fuente: Eviews 10.0 (2019). 
En esta investigación se utilizaron datos de panel, donde todas las variables objeto de estudio fueron estandarizadas con puntuaciones estándar (número de desviaciones estándar que hay por encima o por debajo de la media del ranking e índice de competitividad global publicado por el Foro Económico Mundial). ${ }^{6}$ Por su parte, el PIB per cápita fue estimado usando datos suministrados por la Penn World Table $3 .{ }^{7}$ Los resultados obtenidos fueron los siguientes:

Tabla 3. Análisis de confiabilidad

\begin{tabular}{|c|l|c|c|c|c|}
\hline \multicolumn{3}{|c|}{ Resumen de procesamiento de casos } & \multicolumn{2}{c|}{ Estadísticas de fiabilidad } \\
\hline \multirow{4}{*}{ Casos } & N & $\%$ & Alfa de Cronbach & N de Elementos \\
\cline { 2 - 4 } & Válido & 240 & 100,0 & \multirow{2}{*}{0,931} & 5 \\
\cline { 2 - 4 } & Excluido $^{\mathrm{a}}$ & 0 & 0,0 & & \\
\cline { 2 - 4 } & Total & 240 & 100,0 & & \\
\hline
\end{tabular}

a. La eliminación por lista se basa en todas las variables del procedimiento.

Fuente: SPSS Ver. 24.0

El análisis de fiabilidad se efectuó realizando la estimación del coeficiente de Alfa Cronbach de las variables previamente estandarizadas con las funciones Z. En este sentido, la confiabilidad o fiabilidad, representa a la estabilidad o persistencia de la evolución de una variable respecto a diferentes sujetos u objetos de investigación (Kerlinger \& Lee, 2002). En este caso las variables son las puntuaciones $\mathrm{Z}$ del índice y ranking de competitividad y libertad económica y PIB per cápita y los objetos de investigación son las economías sujetas a estudio.

El coeficiente de Alfa Cronbach de 0,931 indica que la data sujeta a estudio es 93,1\% confiable, es decir existe un alto grado de asociación o correlación lineal entre las sumas de las varianzas de las puntaciones $Z Z=\frac{X_{i}-\bar{X}}{\sigma}$ na de las 20 eco nomías sujetas a estudios, respecto a la suma de ias varianzas de cada variable respecto a los 240 casos analizados.

Tabla 4. Análisis de clúster

\begin{tabular}{|l|c|c|c|c|}
\hline \multicolumn{5}{|c|}{ Centros de clústeres finales } \\
\hline \multirow{4}{*}{\begin{tabular}{l} 
Puntuación Z de PIB per cápita \\
\multirow{2}{*}{$\begin{array}{l}\text { Clúster de pertenencia de acuerdo } \\
\text { nivel de PIB per cápita }\end{array}$}
\end{tabular}} & 1 (Bajo) & 2 (Moderado) & 3 (Alto) & 4 (Muy alto) \\
\cline { 2 - 6 } & $-0,78$ & $-0,30$ & 0,22 & 2,71 \\
\cline { 2 - 6 } & $\$ 5.399$ & $\$ 11.047$ & $\$ 17.241$ & $\$ 46.698$ \\
\hline Casos & 1 (Bajo) & 2 (Moderado) & 3 (Alto) & 4 (Muy alto) \\
\hline
\end{tabular}

Fuente: SPSS Ver. 24.0

6 Publicado anualmente desde 1979 abarca 140 países; el índice y ranking de libertad económica creado en el año 1995, incluye a 180 naciones, y publicado desde entonces anualmente por La Fundación Heritage y el Wall Street Journal.

$7 \quad$ Es un conjunto de datos de cuentas nacionales desarrollado y mantenido por académicos de la Universidad de California, y el Centro de Desarrollo de Crecimiento Groningen de la facultad de economía y negocios de la Universidad de Groningen, la misma es una base de datos con información sobre niveles relativos de ingresos, productos, insumos y productividad, que abarca 182 países entre 1950 y 2017 en función la paridad del poder compra. 
Ahora bien, en esta investigación se empleó la técnica de aglomeración de k-medias, la cual según Everitt y Hothorn (2011) pretende dividir los n sujetos u objetos de estudio en un conjunto de datos multivariados en k grupos o agrupaciones, $(\mathrm{G} 1, \mathrm{G} 2, \ldots$, Gk), donde denota el conjunto de sujetos u objetos de estudio en el grupo i, y se da k a una posible variable que el investigador especifica el rango, minimizando algún criterio numérico, donde los valores bajos de que se consideran indicativos dé una "buena" solución. La más utilizada es la implementación de k-means clustering (clúster de k-medias), la cual desea hallar la partición de los $\mathrm{n}$ sujetos en $\mathrm{k}$ grupos que reduce la suma dentro de grupo de cuadrados (WGSS) sobre todas las variables; explícitamente, este criterio es:

$$
W G S S=\sum_{j=1}^{q} \sum_{l=1}^{k} \sum_{i \in G_{i}}\left(X_{i j}-\overline{X_{j}^{(I)}}\right)^{2}
$$

Donde $\bar{X}_{i j}^{(l)}\llcorner$ media de los elementos en el grupo en la variable.

Estableciendo para este caso cuatro conglomerados como se muestra en la Tabla 4: Grupo 1 con 68 casos cuyas economías en promedio tienen un PIB per cápita de \$5399 dólares; el grupo 2 \$11 047 con 85 casos; el grupo 3 con \$17 241 y 63 casos; y el grupo 4 con un PIB per cápita promedio de $\$ 46698$ que se presentan solo 24 casos resumidos en los que destacan por América las economías de Canadá y Estados Unidos de América. Así mismo a continuación se muestra en un índice de valoración de competitividad, libertad económica y crecimiento económico construido por los investigadores de acuerdo con la categorización de las variables sujetas a estudio en una escala del 1 al 5 por los 12 años estudiados para cada economía, cuya suma acumulada máxima tiene una puntuación de 300 puntos y mínima 60 puntos.

\section{Gráfico 1. Estratificación de las economías en el continente americano}

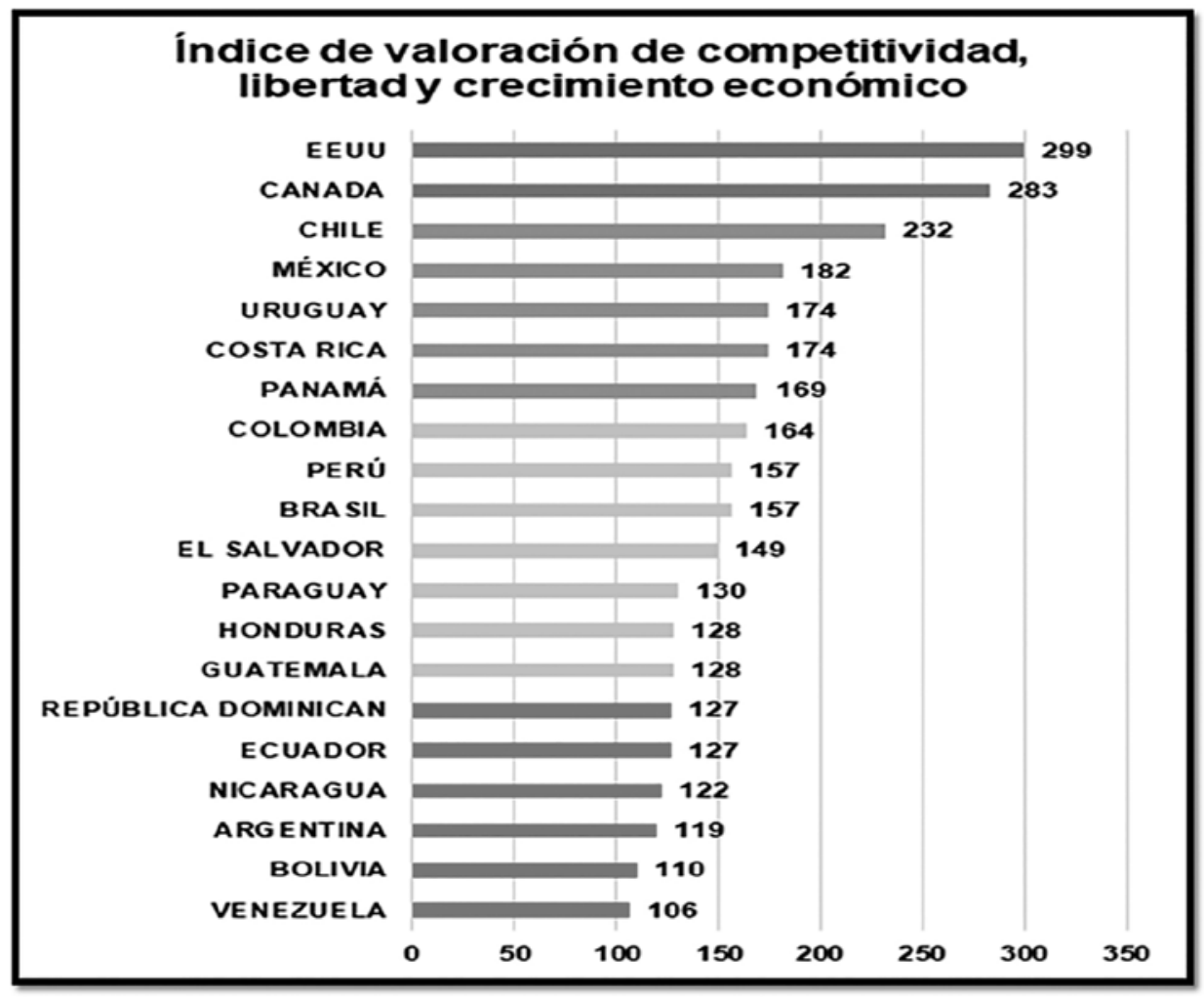

Fuente: Elaboración propia. 
Clúster 4: Estados Unidos y Canadá.

Clúster 3: Chile, México, Uruguay, Costa Rica y Panamá.

Clúster 2: Colombia, Perú, Brasil, El Salvador, Paraguay, Honduras y Guatemala.

Clúster 1: Ecuador, Nicaragua, Argentina, Bolivia y Venezuela.

\subsection{Análisis factorial}

En el determinante de la matriz de coeficientes de correlación, la prueba de contraste de esfericidad de Bartlett de las puntuaciones $\mathrm{Z}$ de las variables estudiadas se aproxima a cero $\Delta_{\mathrm{D}}=0,000173763$ lo cual es un importante indicador para avalar la utilización del análisis factorial, porque denota un alto grado de asociación lineal entre las variables consideradas (ver Tabla 5).

\section{Tabla 5. Matriz de Correlaciones}

\begin{tabular}{|c|c|c|c|c|c|c|c|}
\hline \multicolumn{8}{|c|}{ Matriz de correlaciones ${ }^{a}$} \\
\hline & & 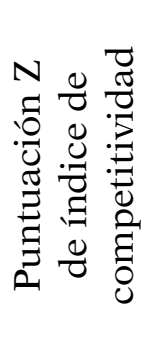 & 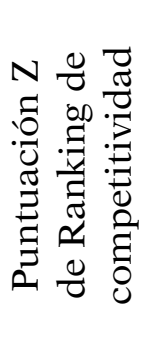 & 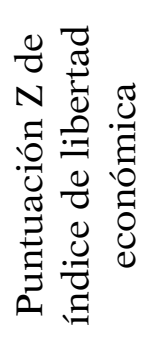 & 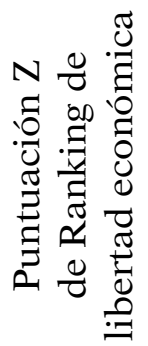 & 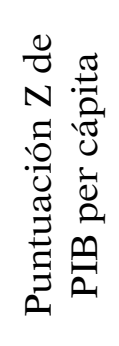 & $\frac{\bar{v}}{\stackrel{\tilde{D}}{\mathscr{U}}}$ \\
\hline \multirow{6}{*}{ Correlación } & $\begin{array}{l}\text { Puntuación } \mathrm{Z} \text { de Índice de } \\
\text { competitividad }\end{array}$ & 1,000 & 0,963 & 0,661 & 0,704 & 0,886 & 0,750 \\
\hline & $\begin{array}{l}\text { Puntuación Z de Ranking } \\
\text { de competitividad }\end{array}$ & 0,963 & 1,000 & 0,671 & 0,744 & 0,794 & 0,719 \\
\hline & $\begin{array}{l}\text { Puntuación } \mathrm{Z} \text { de Índice de } \\
\text { libertad económica }\end{array}$ & 0,661 & 0,671 & 1,000 & 0,902 & 0,468 & 0,323 \\
\hline & $\begin{array}{l}\text { Puntuación Z de Ranking } \\
\text { de libertad económica }\end{array}$ & 0,704 & 0,744 & 0,902 & 1,000 & 0,509 & 0,395 \\
\hline & $\begin{array}{l}\text { Puntuación Z de PIB per } \\
\text { cápita }\end{array}$ & 0,886 & 0,794 & 0,468 & 0,509 & 1,000 & 0,861 \\
\hline & clúster & 0,750 & 0,719 & 0,323 & 0,395 & 0,861 & 1,000 \\
\hline
\end{tabular}

a. Determinante $=, 000$

Fuente: SPSS Ver. 24.0

Tabla 6. Prueba de suficiencia muestral KMO

\begin{tabular}{|l|l|c|}
\hline \multicolumn{2}{|c|}{ Prueba de KMO y Bartlett } \\
\hline \multirow{3}{*}{ Medida Kaiser-Meyer-Olkin de adecuación de muestreo } & 0,690 \\
\hline \multirow{3}{*}{ Prueba de esfericidad de Bartlett } & Aprox. Chi-cuadrado & 2044,687 \\
\cline { 2 - 3 } & Gl & 15 \\
\cline { 2 - 3 } & Sig. & 0,000 \\
\hline
\end{tabular}

Fuente: SPSS Ver. 24.0 
La medida adecuación del muestreo general (Kaiser-Mayer-Olkin) que se muestra en la Tabla 6 es una medida global indicativa de si se lleva a cabo el análisis de factores, ¿qué tan fuerte y adecuada sería la posible solución a encontrar? Mientras más grande es este valor, la solución es más fuerte; lo óptimo es que sea: MASg $\geq 0,5$. Y considerada aceptable de acuerdo con Garza et al. (2013). En el caso de este estudio el valor del coeficiente es 0,69 lo cual es aceptable, por lo cual valida la pertinencia del análisis de factores.

Tabla 7. Matriz anti-imagen para medidas de adecuación de muestreo

\begin{tabular}{|c|c|c|c|c|c|c|c|}
\hline \multicolumn{8}{|c|}{ Matrices anti-imagen } \\
\hline & & 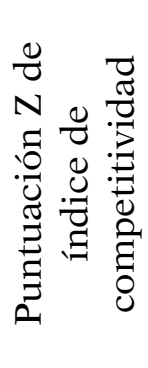 & 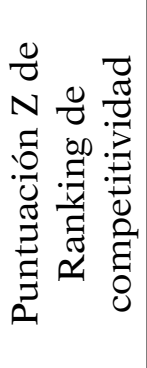 & 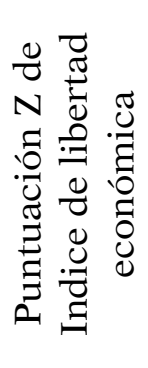 & 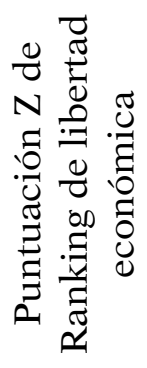 & 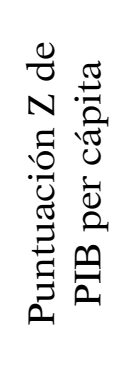 & 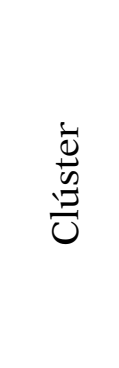 \\
\hline \multirow{6}{*}{$\begin{array}{l}\text { Correlación } \\
\text { anti-imagen }\end{array}$} & $\begin{array}{l}\text { Puntuación } \mathrm{Z} \text { de índice } \\
\text { de competitividad }\end{array}$ &, $665^{a}$ & $-0,894$ & $-0,250$ & 0,187 & $-0,761$ & 0,369 \\
\hline & $\begin{array}{l}\text { Puntuación Z de Ran- } \\
\text { king de competitividad }\end{array}$ & $-0,894$ &, $676^{\mathrm{a}}$ & 0,216 & $-0,339$ & 0,577 & $-0,428$ \\
\hline & $\begin{array}{l}\text { Puntuación } \mathrm{Z} \text { de índice } \\
\text { de libertad económica }\end{array}$ & $-0,250$ & 0,216 &, $724^{\mathrm{a}}$ & $-0,806$ & 0,092 & 0,078 \\
\hline & $\begin{array}{l}\text { Puntuación } \mathrm{Z} \text { de Ran- } \\
\text { king de libertad econó- } \\
\text { mica }\end{array}$ & 0,187 & $-0,339$ & $-0,806$ &, $739^{\mathrm{a}}$ & $-0,067$ & 0,056 \\
\hline & $\begin{array}{l}\text { Puntuación } \mathrm{Z} \text { de } \mathrm{PIB} \\
\text { per cápita }\end{array}$ & $-0,761$ & 0,577 & 0,092 & $-0,067$ &, $653^{a}$ & $-0,691$ \\
\hline & Clúster & 0,369 & $-0,428$ & 0,078 & 0,056 & $-0,691$ &, $721^{\mathrm{a}}$ \\
\hline
\end{tabular}

a. Medidas de adecuación de muestreo (MSA)

Fuente: SPSS Ver. 24.0

La matriz anti-imagen para medidas de adecuación de muestreo, mostrado en la Tabla 7, señala en su diagonal principal que todas las variables consideradas son pertinentes para el análisis de factores, ya que los coeficientes de correlación presentan valores por encima a 0,5. Así mismo, la matriz de comunalidades, que muestra el porcentaje de la varianza total explicada por los factor(es), como se evidencia en la Tabla 8 , indica que los mismos explican el 92,3\% de la variable puntuación $\mathrm{Z}$ del PIB per cápita; el 94,4\% de la variable puntuación $\mathrm{Z}$ del índice de competitividad; el 93,6\% de la variable puntuación $\mathrm{Z}$ del índice de libertad económica. 
Tabla 8. Matriz de comunalidades

\begin{tabular}{|c|c|c|}
\hline \multicolumn{3}{|l|}{ Comunalidades } \\
\hline & Inicial & Extracción \\
\hline Puntuación Z de Índice de competitividad & 1,000 & 0,944 \\
\hline Puntuación Z de Ranking de competitividad & 1,000 & 0,907 \\
\hline Puntuación Z de Índice de libertad económica & 1,000 & 0,936 \\
\hline Puntuación Z de Ranking de libertad económica & 1,000 & 0,940 \\
\hline Puntuación $\mathrm{Z}$ de PIB per cápita & 1,000 & 0,923 \\
\hline Clúster de pertenencia de acuerdo nivel de PIB per cápita & 1,000 & 0,896 \\
\hline
\end{tabular}

Fuente: SPSS Ver. 24.0

Tabla 9. Varianza total de las variables sujetas a estudio explicada por los factores

\begin{tabular}{|c|c|c|c|c|c|c|c|c|c|}
\hline \multirow[b]{3}{*}{ Componente } & \multicolumn{8}{|c|}{ Varianza total explicada } & \\
\hline & \multicolumn{3}{|c|}{ Autovalores iniciales } & \multicolumn{3}{|c|}{$\begin{array}{l}\text { Sumas de extracción } \\
\text { de cargas al cuadrado }\end{array}$} & \multicolumn{3}{|c|}{$\begin{array}{l}\text { Sumas de extracción } \\
\text { de cargas al cuadrado }\end{array}$} \\
\hline & 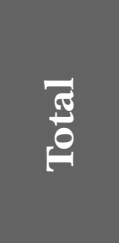 & 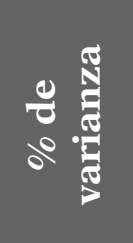 & de & 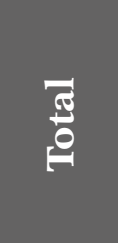 & 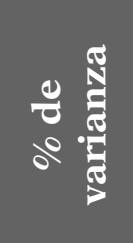 & o & లే & 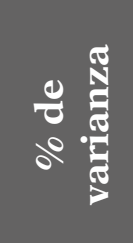 & de \\
\hline 1 & 4,483 & 74,725 & 74,725 & 4,483 & 74,725 & 74,725 & 3,044 & 50,737 & 50,737 \\
\hline 2 & 1,062 & 17,705 & 92,430 & 1,062 & 17,705 & 92,430 & 2,502 & 41,693 & 92,430 \\
\hline 3 & 0,215 & 3,582 & 96,012 & & & & & & \\
\hline 4 & 0,141 & 2,353 & 98,364 & & & & & & \\
\hline 5 & 0,084 & 1,396 & 99,761 & & & & & & \\
\hline 6 & 0,014 & 0,239 & 100,000 & & & & & & \\
\hline
\end{tabular}

Fuente: SPSS Ver. 24.0

En la Tabla 9 se muestra cómo los dos factores considerados explican el 92,43\% de la varianza total de las variables sujetas a estudio. El primer factor explica el 74,73\% y el segundo factor el $17,71 \%$. 


\section{Tabla 10. Matriz de coeficiente de puntuación de componente y componente rotado}

\begin{tabular}{|c|c|c|c|c|c|}
\hline \multicolumn{3}{|c|}{$\begin{array}{l}\text { Matriz de coeficiente de } \\
\text { puntuación de componente }\end{array}$} & \multicolumn{3}{|c|}{ Matriz de componente rotadoa } \\
\hline & \multicolumn{2}{|c|}{ Componente } & & \multicolumn{2}{|c|}{ Componente } \\
\hline & 1 & 2 & & 1 & 2 \\
\hline $\begin{array}{l}\text { Puntuación } \mathrm{Z} \text { de índice de } \\
\text { competitividad }\end{array}$ & 0,227 & 0,066 & $\begin{array}{l}\text { Puntuación } \mathrm{Z} \text { de índice de } \\
\text { competitividad }\end{array}$ & 0,802 & \\
\hline $\begin{array}{l}\text { Puntuación } Z \text { de Ranking } \\
\text { de competitividad }\end{array}$ & 0,178 & 0,119 & $\begin{array}{l}\text { Puntuación } \mathrm{Z} \text { de Ranking } \\
\text { de competitividad }\end{array}$ & 0,741 & \\
\hline $\begin{array}{l}\text { Puntuación } \mathrm{Z} \text { de índice de } \\
\text { libertad económica }\end{array}$ & $-0,229$ & 0,532 & $\begin{array}{l}\text { Puntuación } \mathrm{Z} \text { de índice de } \\
\text { libertad económica }\end{array}$ & & 0,946 \\
\hline $\begin{array}{l}\text { Puntuación } \mathrm{Z} \text { de Ranking } \\
\text { de libertad económica }\end{array}$ & $-0,182$ & 0,494 & $\begin{array}{l}\text { Puntuación } \mathrm{Z} \text { de Ranking } \\
\text { de libertad económica }\end{array}$ & & 0,929 \\
\hline $\begin{array}{l}\text { Puntuación } \mathrm{Z} \text { de PIB per } \\
\text { cápita }\end{array}$ & 0,386 & $-0,151$ & $\begin{array}{l}\text { Puntuación } \mathrm{Z} \text { de PIB per } \\
\text { cápita }\end{array}$ & 0,921 & \\
\hline Clúster & 0,456 & $-0,265$ & Clúster & 0,940 & \\
\hline \multicolumn{3}{|c|}{$\begin{array}{l}\text { Método de extracción: análisis de } \\
\text { componentes principales. } \\
\text { Método de rotación: Varimax con } \\
\text { normalización Kaiser. } \\
\text { Puntuaciones de componente. }\end{array}$} & \multicolumn{3}{|c|}{$\begin{array}{l}\text { Método de extracción: análisis de } \\
\text { componentes principales. } \\
\text { Método de rotación: Varimax con } \\
\text { normalización Kaiser. } \\
\text { Puntuaciones de componente. }\end{array}$} \\
\hline
\end{tabular}

Fuente: SPSS Ver. 24.0

$\mathrm{F}_{2}=0,07 \mathrm{PZIC}+0,12 \mathrm{PZRC}+0,53 \mathrm{PZILE}+0,49 \mathrm{PZRLE}-0,15 \mathrm{PZPIBPC}-0,27 \mathrm{CNPIB}$

$\mathrm{F}_{2}=0,07 \mathrm{PZIC}+0,12 \mathrm{PZRC}+0,53 \mathrm{PZILE}+0,49 \mathrm{PZRLE}-0,15 \mathrm{PZPIBPC}-0,27 \mathrm{CNPIB}$

La matriz de coeficiente de componente muestra los estimadores insesgados de los factores $F_{1}$ y $F_{2}$ con base en las puntuaciones $\mathrm{Z}$ de cada variable sujeta a estudio, tal como se muestra en la Tabla 10 y las ecuaciones 1 y 2 . Así mismo, en la Tabla 10, se observa la matriz de los componentes rotados la cual nos indica qué variables son explicadas en mayor cuantía por cada factor, en este caso, las variables relacionadas con la competitividad y el crecimiento económico son explicadas por el factor 1; mientras las variables relacionadas con la libertad económica son explicadas por el factor 2 . Se procedió con la estimación de un modelo de regresión múltiple utilizando como variable dependiente el PIB per cápita a nivel, obteniendo los siguientes resultados en las siguientes tablas:

\section{Tabla 11. Coeficiente de correlación y determinación entre la variable real y el pronóstico}

\begin{tabular}{|c|l|c|c|c|}
\hline \multicolumn{3}{|c|}{ Resumen del modelo } \\
Modelo & $\mathrm{R}$ & R cuadrado & R cuadrado ajustado & Error estándar de la estimación \\
\hline 1 &, $951^{\mathrm{a}}$ & 0,905 & 0,904 & 3656,53356 \\
\hline
\end{tabular}

a. Predictores: (Constante), REGR factor score 2 for analysis 3, REGR factor score 1 for analysis 3

b. Variable dependiente: PIB_PER_CAPITA

Fuente: SPSS Ver. 24.0 
El coeficiente correlación lineal de Pearson expresa que el porcentaje de asociación lineal entre la variable real y pronosticada es de $95,1 \%$; el $\mathrm{R}^{2}$ muestra que el porcentaje de la varianza de la variable dependiente explicado por la regresión es de $90,5 \%$ y el $\mathrm{R}^{2}$ (ajustado) apunta a que $90,4 \%$ de la varianza de la variable dependiente es explicado por las variables independientes de manera conjunta.

\section{Tabla 12. Coeficiente de correlación y determinación entre la variable real y el pronóstico}

\begin{tabular}{|c|l|l|l|l|l|l|}
\hline \multicolumn{7}{|c|}{ ANOVA $^{\text {a }}$} \\
\hline \multicolumn{2}{|c|}{ Modelo } & \multicolumn{1}{|c|}{$\begin{array}{c}\text { Suma de } \\
\text { cuadrados }\end{array}$} & \multicolumn{1}{|c|}{ gl } & \multicolumn{1}{c|}{$\begin{array}{c}\text { Media } \\
\text { cuadrática }\end{array}$} & F & Sig. \\
\hline \multirow{3}{*}{1} & Regresión & $30.270 .356 .045,53$ & 2,00 & $15.135 .178 .022,77$ & $1.132,01$ &, $000^{\mathrm{b}}$ \\
\cline { 2 - 8 } & Residuo & $3.168 .746 .332,43$ & 237,00 & $13.370 .237,69$ & & \\
\cline { 2 - 8 } & Total & $33.439 .102 .377,96$ & 239,00 & & & \\
\hline
\end{tabular}

a. Variable dependiente: PIB PER CÁPITA REAL

b. Predictores: (Constante), REGR factor score 2 for analysis 3, REGR factor score 1 for analysis 3

Fuente: SPSS Ver. 24.0

En el análisis de varianza mostrado en la Tabla 12, se evidencia que el modelo es significativo ya que el sig. (Bilateral) $<0,05$ el estadístico $\mathrm{F}$ de Fisher $\mathrm{F}_{\text {(calculado) }}=1.132>$ $\mathrm{F}_{\text {(teórico) }}=26,13$. Al 5\% de significación; por lo que se rechaza Ho, las variables explicativas influyen de forma conjunta y lineal sobre la variable explicada.

\section{Tabla 13. Coeficiente insesgado del modelo de regresión}

\begin{tabular}{|c|c|c|c|c|c|c|c|c|}
\hline \multicolumn{9}{|c|}{ Coeficientes $^{\mathrm{a}}$} \\
\hline & \multirow{2}{*}{$\begin{array}{l}\text { Modelo } \\
\text { B }\end{array}$} & \multicolumn{2}{|c|}{$\begin{array}{l}\text { Coeficientes no } \\
\text { estandarizados }\end{array}$} & \multirow[t]{2}{*}{$\begin{array}{c}\text { Coeficientes } \\
\text { estandarizados }\end{array}$} & \multirow[t]{2}{*}{$t$} & \multirow{2}{*}{$\begin{array}{l}\text { Sig. } \\
\text { Tolerancia }\end{array}$} & \multicolumn{2}{|c|}{$\begin{array}{c}\text { Estadisticas } \\
\text { de } \\
\text { colinealidad }\end{array}$} \\
\hline & & $\begin{array}{l}\text { Error } \\
\text { estándar }\end{array}$ & Beta & & & & VIF & \\
\hline \multirow{3}{*}{1} & (Constante) & $14.642,87$ & 236,03 & & 62,04 & 0,00 & & \\
\hline & $\begin{array}{l}\text { REGR factor score } 1 \\
\text { for analysis } 3\end{array}$ & $10.741,15$ & 236,52 & 0,91 & 45,41 & 0,00 & 1,00 & 1,00 \\
\hline & $\begin{array}{l}\text { REGR factor score } 2 \\
\text { for analysis } 3\end{array}$ & $3.358,86$ & 236,52 & 0,28 & 14,20 & 0,00 & 1,00 & 1,00 \\
\hline
\end{tabular}

a. Variable dependiente: PIB PER CÁPITA REAL

Fuente: SPSS Ver. 24.0

Se puede observar en la Tabla 13, que los estimadores insesgados son significativos al igual que el intercepto, ya que el sig. (Bilateral) < 0,05; así mismo el Factor de inflación de la varianza (FIV) de cada estimador como el de la constante o intercepto de regresión $\mathrm{VIF}<15$, con lo cual no se evidencian problemas de colinealidad entre las variables independientes o explicativas. 
Tabla 14. Prueba de normalidad de los residuos del modelo de regresión

\begin{tabular}{|c|c|c|c|c|c|c|}
\hline \multicolumn{7}{|c|}{ Pruebas de normalidad } \\
\hline & \multicolumn{3}{|c|}{ Kolmogorov-Smirnova } & \multicolumn{3}{|c|}{ Shapiro-Wilk } \\
\hline & Estadístico & gl & Sig. & Estadístico & gl & Sig. \\
\hline RESIDUOS & 0,051 & 240 & ,200* & 0,980 & 240 & 0,002 \\
\hline
\end{tabular}

*. Esto es un límite inferior de la significación verdadera.

a. Corrección de significación de Lilliefors

Fuente: SPSS Ver. 24.0

Finalmente, la prueba de normalidad de los residuos mostrado en la Tabla 14 y Gráfico 2, señala un sig. (Bilateral) >0,05 para la prueba de Kolmogorov-Smirnov sig. (Bilateral) $=0,200$ para muestras mayores a 50 sujetos u objetos, el cual demuestra la existencia de una distribución normal en los residuos del modelo.

\section{Gráfico 2. Histograma de los residuos del modelo de regresión}

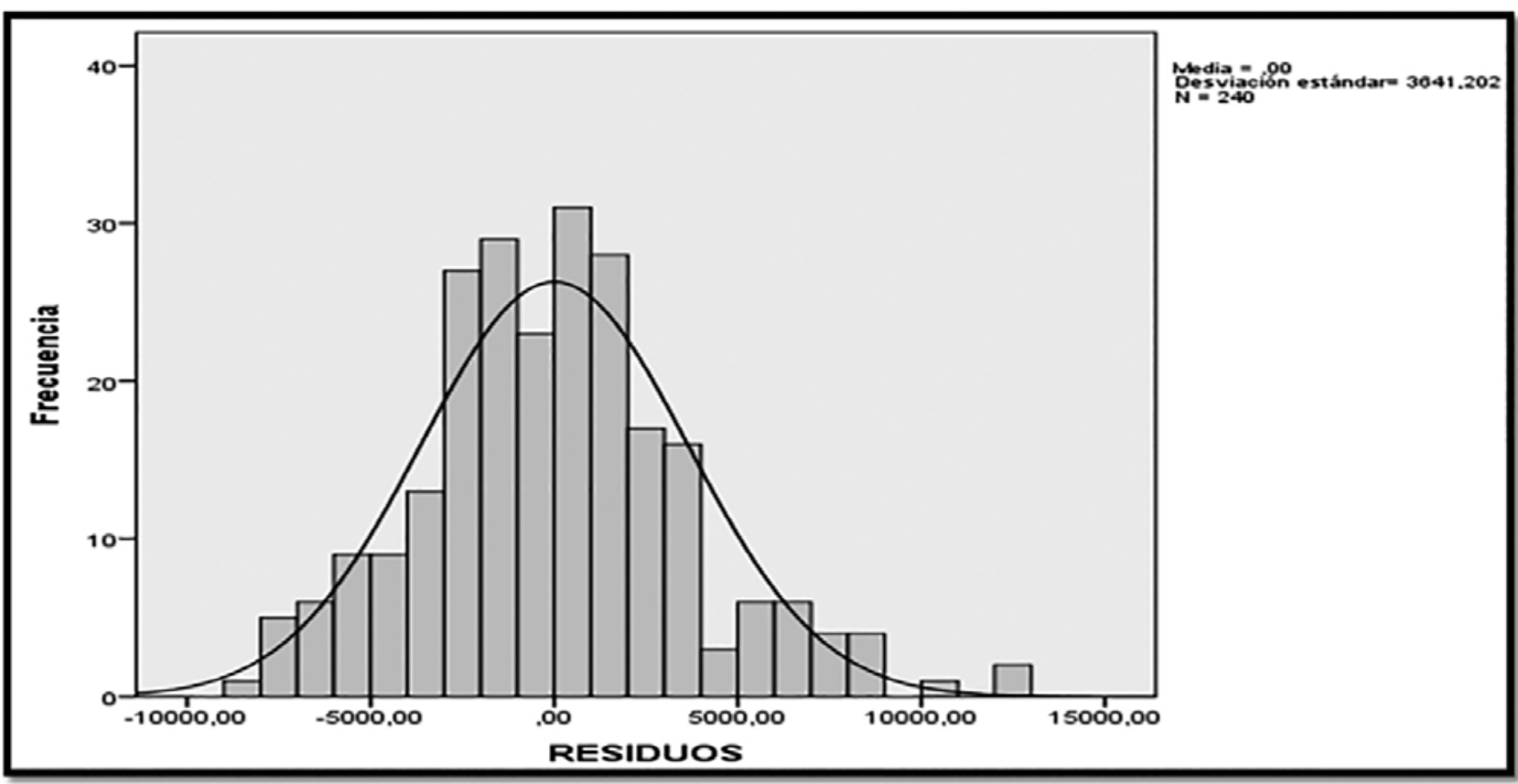

Fuente: SPSS Ver. 24.0

Tabla 15. Prueba de homogeneidad los residuos del modelo de regresión entre clúster

\section{Pruebas de homogeneidad de varianzas}

\begin{tabular}{|c|c|c|c|}
\hline Estadístico de Levene & gl1 & gl2 & Sig. \\
\hline 1,117 & 3 & 236 & 0,343 \\
\hline
\end{tabular}

Fuente: SPSS Ver. 24.0

La Tabla 15, muestra los resultados de estadístico de Levene, en donde se contrasta Ho de homogeneidad de varianzas poblacionales y cuya regla de decisión establece si Sig. (Bilateral) $>0,05$ se rechaza Ho. El resultado arroja que no se rechaza la hipótesis nula. Por lo tanto, existe homogeneidad en las varianzas de los residuos entre clúster. En el 
Gráfico 3, se muestra una vez más la eficiencia del modelo al graficar PIB real promedio de cada país vs. el proyectado, donde uno es prácticamente colineal con el otro.

\section{Gráfico 3. Gráfico del PIB real promedio de cada país vs. el proyectado}

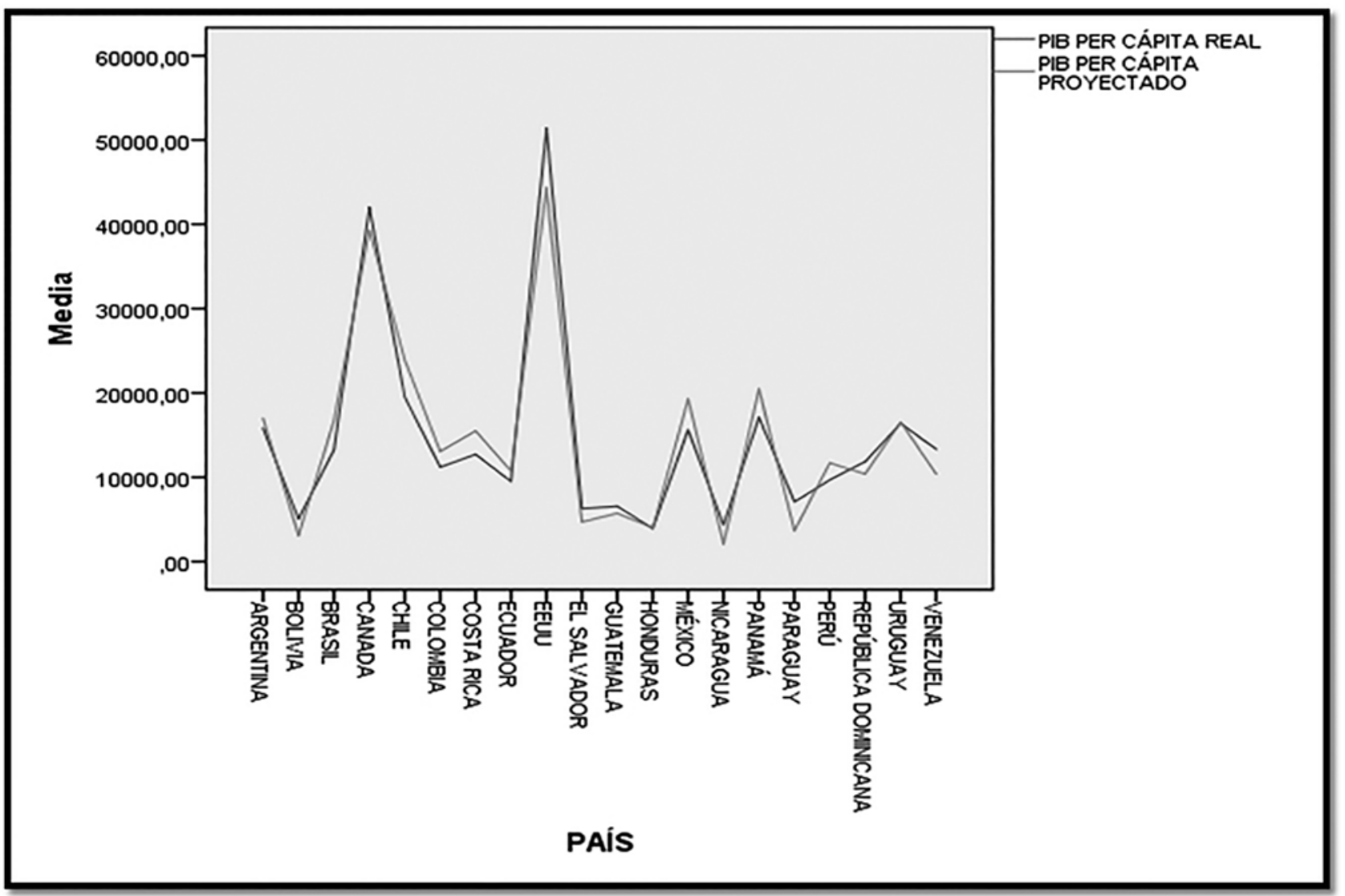

Fuente: SPSS Ver. 24.0

\section{Conclusiones y discusión}

El elemento concluyente más relevante de este estudio es, sin duda, la demostración estadística y econométrica sobre la tesis expuesta por la escuela austriaca de economía según la cual, las economías más libres y competitivas, son aquellas que muestran mayores avances y posicionamiento a nivel internacional en materia de crecimiento económico. Así mismo, se comprobó la relación de causalidad bidireccional entre el índice de libertad económica y el PIB per cápita para cada una de las economías estudiadas en el panel; como el significativo grado de cointegración a largo plazo entre la competitividad, la libertad económica y PIB per cápita.

También es importante destacar cómo la competitividad y el crecimiento económico son explicados estadísticamente con datos disponibles hasta el momento por un factor, mientras la libertad económica es explicada por otro factor. No obstante, ambos factores explican en más de un $90 \%$ la varianza del crecimiento económico medido a través del PIB per cápita. Esto permitió al estudio, construir un modelo de regresión que, más que un pronóstico del PIB per cápita, demostrase de manera más fehaciente la hipótesis planteada en la investigación, según la cual una mayor competitividad y libertad económica se traducen en mayores niveles de crecimiento económico.

Así mismo, es importante enfatizar, cómo en el caso de las economías del continente americano, el factor competitividad tiene una mayor incidencia para explicar el 
crecimiento, que la libertad económica, ello se evidencia en las diferencias significativas en el promedio de PIB per cápita del clúster 4 conformado por Estados Unidos y Canadá respecto al resto de las economías del continente. El contraste se materializa cuando economías como las de Ecuador, Nicaragua, Argentina, Bolivia y Venezuela han terminado cercenando los incentivos de competitividad y libertad económica, muestran menores niveles de crecimiento económico incluso contracción del PIB per cápita. La razón se localiza en: 1) la aplicación de políticas monetarias y fiscales inflacionistas; 2) injerencia en el sistema de precios mediante el establecimiento de controles; 3) una mayor presión fiscal que cubre principalmente déficits fiscales acumulados; y 4) el incremento desproporcionado de las regulaciones estatales.

Las anteriores razones tienen su asidero en las teorías según las cuales para que el sistema capitalista funcione de forma eficiente (corregir los fallos de mercado lograr un reparto más equitativo del ingreso nacional disponible) y ofrecer bienestar social a través del libre mercado, debe tolerar una mayor intervención del gobierno a través de su política fiscal, monetaria, crediticia y presupuestal. Para ello, el sector público no debía temerle a la ejecución de presupuestos desequilibrados financiados con endeudamiento, porque todo ello se traducirá en un aumento de la demanda y oferta agregada interna. En consecuencia, el gobierno, producto de la expansión económica recaudará más impuestos directos e indirectos. No obstante, la realidad es que la política fiscal se hace cada vez más expansiva y el de endeudamiento externo alcanza grados superlativos, mientras la economía crece a tasas muy por debajo del crecimiento de la población con lo cual se genera un empobrecimiento generalizado producto de la caída del PIB per cápita.

\section{Referencias}

Akerlof, G. (1970). The market for lemons: quality, uncertainty and the market mechanism. Quarterly Journal of Economics, 84, 488-500. https://bit.ly/305I24E

Aramburo-González, F. (1998). Turgot. Cuadro filosófico de los progresos sucesivos del espíritu humano y otros textos. Fondo de Cultura Económica.

Aristóteles (1992). Ética Nicomaquea. Ediciones universales.

Baumol, W. (1990). Entrepreneurship: Productive, unproductive, and destructive. The Journal of Political Economy, 98(5), 893-921.

Bunge, M. (1999). Las ciencias sociales en discusión. Una perspectiva filosófica. Traducción de Horacio Pons. Editorial Sudamericana.

Cadavid, J., \& Franco, H. (2006). Factores determinantes de la relación entre el crecimiento económico, la equidad y la competitividad. Ecos de Economía: A Latin American Journal of Applied Economics, 10(23), 105-153. https://bit.ly/3kFFdka

Cantillon, R. (2002[1959]). Essai sur la nature du commerce in Général. Essay on the Nature of Trade in General. Liberty Fund, Inc.

Centro de Estudios sobre la Cuenca del Pacífico (2018). Facultad de Ciencias Económicas y Administrativas de la Pontificia Universidad Javeriana sede Cali-Colombia, Boletín informativo Volumen 3. $\mathrm{N}^{\circ} 4$, diciembre.

Córdova-Pacheco, A. Moina-Sánchez, P., \& Morales-Carrasco, L., \& (2020). Crecimiento económico en una región emprendedora en el Ecuador. Retos Revista de Ciencias de la Administración y Economía, 10(19), 65-80. https://doi.org/10.17163/ret.n19.2020.04

Cuadernos Fundación BBVA (2008). Competitividad y crecimiento: una perspectiva regional. Número 8 sobre capital y crecimiento. Serie disponible en www.fbbva.es.

DeLong, J.B. (2003). Macroeconomía. Editorial McGraw-Hill.

Demmert, H., \& Klein, D. (2003) Experiment on entrepreneurial discovery: an attempt to demonstrate the conjecture of Hayek and Kirzner. Journal of Economic Behavior and Organization, 50, 295-310.

Everitt, B., \& Hothorn, T. (2011). an introduction to applied multivariate analysis with R. First Editions, Edi- 
torial Springer. https://doi.org/10.1007/978-1-4419-9650-3

Fergunson, J. (1979[1938]). Historia de la Economía. Fondo de Cultura Económica. Título original en lengua inglesa: Landmarks of Economic Thought, Longmans, Green and Co., London.

Hayek, F.A von. (1978). Competition as a discovery and procedure. In Friedrich A. Hayek, New studies in Philosophy, Politics, Economics, and the History of ideas (pp. 179-190). University of Chicago Press.

Hayek, F.A von. (1989). The pretence of knowledge. American Economic Review, 79, 3-7.

Garrison, Roger, W. (2001). The macroeconomics of capital structure. Routledge.

Garza-García, J. de la, Morales-Serrano, B.N., \& González-Cavazos, B.A. (2013). Análisis estadístico multivariante. 1a. ed. McGraw-Hill.

Huerta de Soto, J. (2010) La teoría de la eficiencia económica. Revista de economía $\mathcal{E}$ administración, 7(2), julio-diciembre. https://bit.ly/3kDE78I

Kane, E.J. (1981). Accelerating inflation, technological innovation, and the decreasing effectiveness of bank regulation. Journal of Finance, 36, 355-367. https://doi.org/10.1111/j.1540-6261.1981.tb00449.x

Kerlinger, F., \& Lee, H. (2002). Investigación del comportamiento. Métodos de investigación en Ciencias Sociales. McGraw-Hill.

Kirzner, I. (1979). Perception, opportunity and profit. The University of Chicago Press.

Kirzner, I. (1985). The entrepreneurial process. In Israel M. Kirzner (Ed.), Discovery and the capitalist process (pp. 68-93). University of Chicago Press.

Kirzner Israel M. (1997). How markets work disequilibrium, entrepreneurship and discovery. first published in june 1997 second impression april 2000 by the institute of economic affairs 2 Lord North Street Westminster London SW1P3LB.

Knight, F. (1964[1921]) Risk, uncertainty, and profit. Augustus M. Kelley, Bookseller. Reprints of Economic Classics. https://bit.ly/3qezq6v

Leibenstein, H. (1966). Allocative efficiency vs. X-Efficiency. The American Economic Review, 56(3), 392-415. https://bit.ly/3kFmUeU

Leibenstein, H. (1968). Entrepreneurship and development. American Economic Review, 58(2), 72-83. https://bit.ly/30fm4fr

Malthus, R.T. (1977) Ensayo sobre el principio de la población. Primera reimpresión. Fondo de Cultura Económica de México.

Marczyk, G., DeMatteo, D., \& Festinger, D. (2005). Essentials of Research Design and Methodology. John Wiley \& Sons, Inc.

Mill, J.S. (1985[1848]) Principios de Economía Política. Segunda reimpresión. Fondo de Cultura Económica de México.

Mises, L.E. (1983[1944]). Bureaucracy. Libertarian press, Inc.

Morgenstern, O. (1972). Thirteen Critical Points in Contemporary Economic Theory: An Interpretation. Journal of Economic Literature, 10, 1163-1189. https://bit.ly/3beDcbQ

Molero, L., Anchundia, J., Patiño, R., \& Escobar, Y. (2020). Crecimiento económico y apertura comercial: Teoría, datos y evidencia (1960-2017). Revista de Ciencias Sociales (Ve), XXVI(4), 476496. https://doi.org/10.31876/rcs.v26i4.34675

Ricardo, D. (1973). Principios de Economía política y tributación. Segunda reimpresión. Fondo de Cultura Económica.

Rothbard, M. (1979). Comment: The myth of efficiency. In M.J. Rizzo (Ed.), Time, uncertainty and disequilibrium. Lexington Books.

Rothbard, N.M. (2001). Monopolio y competencia. Revista Libertas 34 (mayo 2001) Instituto Universitario ESEADE. https://bit.ly/3sPgTiz

Rothbard, N. M. (2012). Historia del pensamiento económico. Volumen I. Unión Editorial.

Salvat (1972). Enciclopedia Salvat Diccionario. Tomo 5 (Elec-Frai). Salvat Editores.

Samuelson, Paul, P. A., Nordhaus, W. D. y McCallum, J. (1983). Economics. Sexta edición. Toronto y New York: McGraw-Hill, Ryerson.

Say, J.B. (2001[1841]) Tratado de Economía Política. Fondo de Cultura Económica de México. Título 
original en francés: «Traité D'économie politique ou simple exposition de la manière dont se foment se distribuent et se composent les richesses».

Schumpeter, J. (1976[1912]). Teoría del desenvolvimiento económico: una investigación sobre ganancias, capital, crédito, interés y ciclo económico. Cuarta reimpresión. Fondo de Cultura Económica de México.

Schumpeter, J.A. (1994[1954]). Historia del análisis económico. Editorial Ariel, S.A. Título original en lengua inglesa: History of Economics Analysis (Oxford University Press, Inc.).

Schumpeter, J.A. (2008[1942]). Capitalism, socialism and democracy. Harper Collins Publishers.

Smith, A. (1982[1776]) Investigación sobre la naturaleza y causas de la riqueza de las naciones. Tercera reimpresión. Fondo de Cultura Económica.

Traverso P., Baño-Hifóng, M., Samaniego, J. (2017). Influencia de la competitividad en el crecimiento económico. Revista Espacios, 38(23), 16. https://bit.ly/3uQWnQz

Walras, L. (1987[1874]). Elementos de Economía política pura o teoría de la riqueza. Editorial Alianza. Título en lengua francesa: Eléments d'économie Politique Pure ou Théorie de la Richesse Sociale.

Woodford, M. (2008). Convergence in Macroeconomics: Elements of the New Synthesis. Paper for the session "Convergence in Macroeconomics?" at the annual meeting of the American Economic Association, New Orleans in January 4, 2008. 